\title{
A Fractional Order Model for the Transmission Dynamics of Measles with Vaccination
}

\author{
Nnaemeka S. Aguegboh1", Nkem R. Nwokoye2, Netochukwu E. Onyiaji², Ogechi R. Amanso², \\ Dominic 0. Oranugo ${ }^{3}$ \\ ${ }^{1}$ Department of Mathematics, Veritas University, Bwari, Nigeria \\ ${ }^{2}$ Department of Mathematics, University of Nigeria, Nsukka, Nigeria \\ ${ }^{3}$ Department of Mathematics, Nnamdi Azikiwe University, Awka, Nigeria \\ Email: *aguegbohn@veritas.edu.ng, regina.nwokoye@unn.edu.ng, onyiajineto@gmail.com, ogechiamanso@gmail.com, \\ oranugodominic@yahoo.com
}

How to cite this paper: Aguegboh, N.S., Nwokoye, N.R., Onyiaji, N.E., Amanso, O.R. and Oranugo, D.O. (2020) A Fractional Order Model for the Transmission Dynamics of Measles with Vaccination. Open Access Library Journal, 7: e6670.

https://doi.org/10.4236/oalib.1106670

Received: July 27, 2020

Accepted: October 12, 2020

Published: October 15, 2020

Copyright $\odot 2020$ by author(s) and Open Access Library Inc.

This work is licensed under the Creative Commons Attribution International License (CC BY 4.0).

http://creativecommons.org/licenses/by/4.0/

\section{(c) (i) Open Access}

\begin{abstract}
In this paper, the fractional order model was adopted to describe the dynamics of measles and to establish how the virus that causes measles is transmitted as well as how to mitigate the conditions that cause the spread. We showed the existence of the equilibrium states. The threshold parameter of the model was evaluated in terms of parameters in the model using the next generation matrix approach. We provided the conditions for the stability of the disease free and the endemic equilibrium points. Also, the stability of the various equilibrium points was studied. Numerical simulations of the model are presented graphically using Adam-Bashforth Method and the results were interpreted. The result also shows that the use of vaccination is the best way to prevent measles outbreak.
\end{abstract}

\section{Subject Areas}

Dynamical System, Mathematical Analysis, Ordinary Differential Equation

\section{Keywords}

Measles, Vaccination, Fractional-Order Differential Equations, Equilibrium

Points, Stability, Numerical Simulations, Predictor-Corrector Method

\section{Introduction}

Disease is an abnormal condition affecting the body of an organism. It is often construed to be a medical condition associated with specific symptoms and signs. It may be caused by external factors. Childhood diseases are serious infectious diseases and measles is famous among them [1]. Measles is a highly contagious 
viral disease caused by measles virus. It is a childhood disease that rarely occurs in adults [1]. It is spread by coughing and sneezing through close interpersonal contact or direct contact with secretions. Measles is an endemic disease, meaning it has been continually present in a community, and many people develop resistance. In populations not exposed to measles, exposure to the new disease can be devastating. When an individual becomes infected with measles virus, the virus begins to multiply within the cells. Measles can lead to serious complications and death. In May 2015, the journal Science published a report in which researchers found that the measles infection can leave a population at increased risk for mortality from other diseases for two to three years. Once a person has become infected, no specific treatment is available, but supportive approach may improve outcomes [2]. Measles is a vaccine-preventable disease. However, vaccination for children is the key public health strategy to prevent many cases of the disease around the world.

E. F. D. Goufo et al. [3] proposed a fractional SEIR epidemic model for spatial and temporal spread of measles in metapopulations. G. Nazir et al. [4] presented fractional dynamical analysis of measles spread model under vaccination corresponding to nonsingular fractional order derivative while M. Farman et al. [5] analysed and provided a numerical solution of an SEIR epidemic model of measles with non-integer time fractional derivatives by using Laplace Adomian Decomposition Method. The approach presented in this paper differs from those presented and references therein. We present a fractional order SEIR (Susceptible-Exposed-Infected-Removed) model to discuss the dynamics of Measles and also show the impact of vaccination on the population.

This paper is organized as follows: a brief review of the fractional calculus is presented in Section 2 with definitions. Section 3 discusses fractional order models while Section 4 presents model analysis involving equilibrium points and stability. Section 5 is devoted to numerical simulations and discussion of results. Section 6 gives the concluding remarks.

\section{Fractional Order Calculus}

The idea of fractional order calculus is as old as integer order calculus. The complexity and lack of application postponed its progress till a few decades ago. Although a large number of works have been done in modeling the dynamics of epidemiological diseases, it has been restricted to integer-order differential equations. Recently, most of the dynamical systems, based on the integer order calculus, have been modified into the fractional order due to flexibility which can be used to precisely fit the experimental data much better than the integer order modeling [6]. The fractional modeling is an advantageous approach which has been used to study the behavior of diseases because the fractional derivative is a generalization of the integer-order derivative. Most of the vaccination models have been established based on ordinary differential equation (ODEs), but in this work we describe the behavior of these systems by fractional order differen- 
tial equations. There are different definitions of the fractional derivative. Among them, Gruwald-Letnikov, Riemann-Liouville and Caputo's fractional derivatives have been used more than others but they are generally not equivalent [7]. Comparing these three fractional derivatives, it is a fact that Caputo's derivative of a constant is equal to zero, which is not true for the Riemann-Liouville derivative. The main advantage of Caputo's approach is that the initial conditions for fractional differential equations with Caputo derivatives take on the same form as for integer-order differential equations. Having this in mind, we restrict our attention to the Caputo derivative of order $\alpha>0$, which is rather applicable to real world. For the purpose of this research work, we now gather some well-known definitions.

\section{Definition of Terms [7]}

Definition 1 The Caputo Fractional derivative of order $\alpha$ of a function $f: \mathfrak{R}^{+} \rightarrow \mathfrak{R}$ is given by

$$
D_{t}^{\alpha} f(t)=\frac{1}{\Gamma(\alpha-n)} \int_{\alpha}^{t} \frac{f^{(n)}(\tau) \mathrm{d} \tau}{(t-\tau)^{\alpha+1-n}} \quad(n-1<\alpha \leq n)
$$

Definition 2 The formula for the Laplace transform of the Caputo derivative is given by

$$
\int_{0}^{\infty} \mathrm{e}^{-p t}\left\{D_{t}^{\alpha} f(t)\right\} \mathrm{d} t=p^{\alpha} F(p)-\sum_{k=0}^{n-1} p^{\alpha-k-1} f^{(k)}(0), \quad(n-1<\alpha \leq n)
$$

Definition 3 The fractional integral of order $\alpha$ of a function $f: \mathfrak{R}^{+} \rightarrow \mathfrak{R}$ is given by

$$
J^{\alpha}(f(x))=\frac{1}{\Gamma(\alpha)} \int_{0}^{x}(x-t)^{\alpha-1} f(t) \mathrm{d} t, \quad \alpha>0, x>0
$$

Definition 4 The fractional integral of the Caputo fractional derivative of order $\alpha$ of a function $f: \Re^{+} \rightarrow \mathfrak{R}$ is given by

$$
J^{\alpha}\left\{D^{\alpha} f(t)\right\}=f(t)-\sum_{k=0}^{n-1} f^{(k)}(0) \frac{t^{k}}{k !}, \quad t>0
$$

Definition 5 A two-parameter function of the Mittag-Leffler type is defined by the series expansion

$$
E_{\alpha, \beta}(z)=\sum_{k=0}^{\infty} \frac{z^{k}}{\Gamma(\alpha k+\beta)}, \quad(\alpha, \beta>0)
$$

\section{Model Assumptions and Formulation}

The SEIR model is based on the following assumptions:

1) The natural death rates $\mu$ in the classes remain unequal to number of births, so that population size $N$ is realistically not constant. In this model, the population $N(t)$ is divided into four subpopulations: The Susceptible, Exposed. Infectious and Recovered, with sizes denoted by $S(t), E(t), I(t)$, and $R(t)$ respectively. 
2) The only way of entry into the population is through birth and the only way of exit is through death from natural causes or death from measles-related causes.

3) That as the population size $N$ (the density of individuals) increases, so does the contact rate (density dependent transmission).

4) The population mixes homogeneously. That is all individuals are equally likely to be infected by the infectious individuals in a case of contact except those who are immune.

5) The infective rate of an infected individual is proportional to the number of Susceptible, the coefficient of the proportion is a constant $\beta$, so that the total number of new infected at time $t$ is $\beta S(t) I(t)$.

6) That an individual who is infected through direct contact with an infectious individual, on recovery the individual obtains permanent infection-acquired immunity that is an individual cannot be infected again by measles.

7) That an individual who has attended first and second dose of vaccine consecutively receive permanent immunity to measles.

8) That a proportion of the population of newborns is immunized against measles infection. That is, they received the first and second dose of vaccine at the rate of $v \Lambda$ and the rest are susceptible.

9) That first dose of vaccine does not confer lifelong immunity.

10) That all newborns are previously not infective or have not contracted the disease.

11) That people in each compartment have equal natural death rate.

The progression of measles within the total population is simplified to four fractional differential equations. These four equations represent four different groups of people according to their epidemiological state namely: the Susceptible $S(t)$, the Exposed $E(t)$, the Infected $I(t)$, and the Recovered $R(t)$. The Susceptible class, $S(t)$ increases due to births which are not immunized and those who received only the first dose of measles vaccine into the population at the rate of $(1-v) \Lambda, v \in[0,1]$, where we assumed that a proportion, $v$, of newborns received the two doses of vaccine and joined the recovered class at the rate of $v \Lambda$. The Susceptible class $S(t)$, are people that have never come into contact with measles and those who received the first dose of vaccine. When there is a direct contact of a susceptible individual with an infectious individual so that transmission occurs at a rate $\beta$, then the Susceptible join the Exposed class, $E(t)$. Exposed class consists of individuals having the disease but do not have the capability to transmit the disease. At the end of incubation period (latent period), exposed individuals will progress to the infectious class, $I(t)$, at a constant rate, $\eta$. Infectious class is those having the disease and is able to transfer it to others. After some treatment, therapy or a certain period of time some infectious individuals will recover at a rate constant, $\varepsilon$. Since the disease is deadly, some infected individuals may die due to the disease at the rate, $\delta$. The Recovered class, $R(t)$, consists of those with permanent infection-acquired immunity and those who received the second dose of vaccine. The individuals are immune for the 
rest of their lives. In addition, natural mortality claims individuals at a rate $\mu$ in each compartment. As most mothers have been infected, IgG antibodies transferred across the placenta, to newborn infants give them temporary passive immunity to measles infection. The flow diagram of the model is as follows (Figure 1):

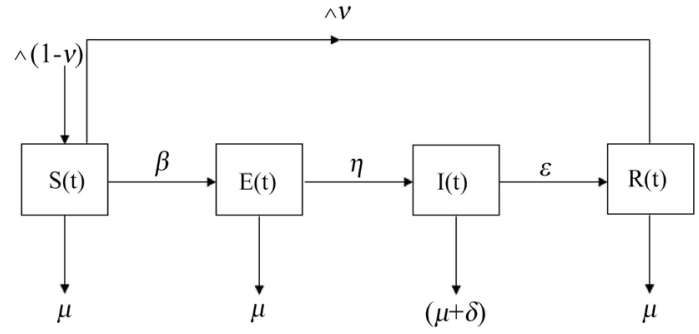

Figure 1. Model flow diagram.

Taking into account the above descriptions and assumptions, the Fractional SEIR model is described by

$$
\left\{\begin{array}{l}
D^{\alpha} S(t)=\Lambda(1-v)-\beta S I-\mu S \\
D^{\alpha} E(t)=\beta S I-\eta E-\mu E \\
D^{\alpha} I(t)=\eta E-\varepsilon I-\delta I-\mu I \\
D^{\alpha} R(t)=\Lambda v+\varepsilon I-\mu R
\end{array}\right.
$$

By setting $\alpha=1$, the system of Equation (3.6) can be reduced to integer order system.

With the non-negative initial condition:

$$
\begin{aligned}
& S(0)=S_{0}, E(0)=E_{0}, I(0)=I_{0}, R(0)=R_{0}, \\
& N=S+E+I+R,(S, E, I, R) \in R_{+}^{4}
\end{aligned}
$$

By adding the four equations of the above system (3.6), we obtain

$$
D^{\alpha} N(t)=\Lambda-\mu N(t)-\delta I
$$

But at DFE, no disease, hence $\delta=0$, so we have:

$$
D^{\alpha} N(t)=\Lambda-\mu N(t)
$$

Lemma 3.1 The closed set $\Omega=\left\{(S, E, I, R) \in R_{+}^{4}: S+E+I+R \leq \frac{\Lambda}{\mu}\right\}$ is positively invariant with respect to model(3.6).

$\underline{\text { Proof }}$

The fractional derivative of the total human population, obtained by adding all the human equations of model (3.6), is given by

$$
D^{\alpha} N(t)=\Lambda-\mu N(t)
$$

Taking the Laplace transform of (3.7) gives:

$$
\begin{aligned}
& S^{\alpha} N(s)-S^{\alpha-1} N(0)=\frac{\Lambda}{S}-\mu N(s) \\
\Rightarrow & N(s)=\frac{\Lambda}{S\left(S^{\alpha}+\mu\right)}+\frac{S^{\alpha-1}}{s^{\alpha}+\mu} N(0)
\end{aligned}
$$


Taking the inverse Laplace transform of (3.8), we have:

$$
N(t)=N(0) E_{\alpha, 1}\left(-\mu t^{\alpha}\right)+\Lambda t^{\alpha} E_{\alpha, \alpha+1}\left(-\mu t^{\alpha}\right)
$$

where $E_{\alpha, \beta}$ is the Mittag-Leffler function. But the fact that the Mittag-Leffler function has an asymptotic behavior [7] [8], it follows that:

$$
\begin{gathered}
E_{\alpha, 1} N(t)=\sum_{k=0}^{\infty} \frac{N^{K}(t)}{\Gamma(\alpha k+1)}, \alpha>0 \\
E_{\alpha, \alpha+1} N(t)=\sum_{k=0}^{\infty} \frac{N^{K}(t)}{\Gamma(\alpha k+\alpha+1)}, \alpha>0
\end{gathered}
$$

Expanding (3.10), we have

$$
E_{\alpha, 1} N(t)=\frac{1}{\Gamma 1}+\frac{N(t)}{\Gamma(\alpha+1)}+\frac{N^{2}(t)}{\Gamma(2 \alpha+1)}+\cdots
$$

Expanding (3.11), we have

$$
E_{\alpha, \alpha+1} N(t)=\frac{1}{\Gamma(\alpha+1)}+\frac{N(t)}{\Gamma(2 \alpha+1)}+\frac{N^{2}(t)}{\Gamma(3 \alpha+1)}+\cdots
$$

Since Mittag-Leffler function has an asymptotic property, we have

$$
N(t)=1+O(N)
$$

Taking limit as $k \rightarrow \infty$, we have

$$
N(t) \approx 1
$$

Then, it is clear that $\Omega$ is a positive invariant set. Therefore, all solutions of the model with initial conditions in $\Omega$ remain in $\Omega$ for all $t>0$. Then, $\Omega=N(t)>0$ implies that it is feasible with respect to model (3.6).

\section{Model Analysis}

\subsection{The Basic Reproduction Number, $\boldsymbol{R}_{0}$}

In many disease transmission models, the peak prevalence of infected hosts and the final size of the epidemic are increasing functions of $R_{0}$, making it a useful measure of spread [9]. Diekmann et al. [10] defined the basic reproduction number $R_{0}$ as the average number of secondary infections caused by an infectious individual during his or her entire life as an infectious person. A paper by [11] provided a method for calculating $R_{0}$, which is the formation of the next-generation matrix. It is comprised of two parts: $F$ and $V$, where

$$
F=\left|\frac{\partial f_{i} x_{(0)}}{\partial x_{j}}\right|, \quad V=\left|\frac{\partial v_{i} x_{(0)}}{\partial x_{j}}\right|
$$

$F_{i}$ is the new infections, while $V_{i}$ is transfer of infection from one compartment to another. $x_{0}$ is the disease free equilibrium point. $R_{0}$ is the spectral radius of the next generation matrix, which is the dominant eigenvalue of the same matrix. To calculate this, we consider the infected compartments $E(t)$ and $I(t)$.

$$
\begin{aligned}
& D^{\alpha} E(t)=\beta S I-\eta E-\mu E \\
& D^{\alpha} I(t)=\eta E-\varepsilon I-\delta I-\mu I
\end{aligned}
$$


Define:

$$
\begin{gathered}
F_{i}=\left(\begin{array}{c}
\beta S I \\
0
\end{array}\right) \\
V_{i}=\left(\begin{array}{c}
(\eta+\mu) E \\
(\varepsilon+\mu+\delta) I-\eta E
\end{array}\right)
\end{gathered}
$$

Therefore, the dominant eigenvalue of $F V^{-1}$ is given as:

$$
R_{0}=\frac{\eta \Lambda \beta(1-v)}{\mu(\eta+\mu)(\varepsilon+\mu+\delta)}
$$

The biological interpretation of $R_{0}$ is that the disease will invade the population if $R_{0}$ exceeds 1 and will die off if $R_{0}$ is less than 1 [12].

\subsection{Equilibrium Points and Their Local Asymptotic Stability}

An equilibrium point is a point at which variables of a system remain unchanged over time. The equilibrium point of system (3.6) satisfies the following system:

$$
D^{\alpha} S(t)=D^{\alpha} E(t)=D^{\alpha} I(t)=D^{\alpha} R(t)=0
$$

such that system (3.6) becomes

$$
\left\{\begin{array}{l}
0=\Lambda(1-v)-\beta S I-\mu S \\
0=\beta S I-\eta E-\mu E \\
0=\eta E-\varepsilon I-\delta I-\mu I \\
0=\Lambda v+\varepsilon I-\mu R
\end{array}\right.
$$

\subsubsection{Stability of the Disease-Free Equilibrium State}

Theorem 4.1 The disease free equilibrium point is locally asymptotically stable if $R_{0}<1$ and unstable if $R_{0}>1$ [12].

Proof. We evaluated the disease free equilibrium points as:

$$
E^{0}=\left(S^{0}, E^{0}, I^{0}, R^{0}\right)=\left(\frac{\Lambda(1-v)}{\mu}, 0,0,0\right)
$$

The Jacobian matrix at disease free equilibrium is given as:

$$
J\left(E_{0}\right)=\left(\begin{array}{cccc}
-\mu & 0 & \frac{\beta \Lambda(1-v)}{\mu} & 0 \\
0 & -(\eta+\mu) & \frac{\beta \Lambda(1-v)}{\mu} & 0 \\
0 & \eta & -(\varepsilon+\mu+\text { delta }) & 0 \\
0 & 0 & \varepsilon & -\mu
\end{array}\right)
$$

The characteristic equation becomes

$$
\left|J\left(E_{0}\right)-I \lambda\right|=\left[\begin{array}{cccc}
-\mu-\lambda & 0 & \frac{\beta \Lambda(1-v)}{\mu} & 0 \\
0 & -(\eta+\mu)-\lambda & \frac{\beta \Lambda(1-v)}{\mu} & 0 \\
0 & \eta & -(\varepsilon+\mu+\text { delta })-\lambda & 0 \\
0 & 0 & \varepsilon & -\mu-\lambda
\end{array}\right]=0
$$


Then, we have

$\lambda_{1,2}=-\mu($ twice $)$, and the characteristic equation is

$$
\lambda^{2}+\lambda(a+b)+a b-c=0
$$

where

$$
\begin{gathered}
a=\eta+\mu>0 \\
b=\varepsilon+\mu+\delta>0 \\
c=\frac{\beta \eta \Lambda(1-v)}{\mu}
\end{gathered}
$$

Now $a b-c=a b\left(1-R_{0}\right)$ and hence, is positive when $R_{0}<1$. By Routh-Hourwitz criterion, the eigenvalues have negative real parts. Therefore, the disease-free equilibrium is locally asymptotically stable if $R_{0}<1$ and unstable if $R_{0}>1$.

\subsubsection{Stability Analysis of the Endemic Equilibrium Point}

The Jacobiam matrix of the system (3.6) is given as

$$
J=\left(\begin{array}{cccc}
-(\beta I+\mu) & 0 & -\beta S & 0 \\
\beta I & -(\eta+\mu) & \beta S & 0 \\
0 & \eta & -(\varepsilon+\mu+\delta) & 0 \\
0 & 0 & \varepsilon & -\mu
\end{array}\right)
$$

At Endemic Equilibrium Point, we have

$$
J\left(E^{*}\right)=\left(\begin{array}{cccc}
-\left(\beta I^{*}+\mu\right) & 0 & -\beta S^{*} & 0 \\
\beta I^{*} & -(\eta+\mu) & \beta S^{*} & 0 \\
0 & \eta & -(\varepsilon+\mu+\delta) & 0 \\
0 & 0 & \varepsilon & -\mu
\end{array}\right)
$$

where

$$
\begin{gathered}
S^{*}=\frac{(\eta+\mu)(\varepsilon+\mu+\delta)}{\eta \beta} \\
E^{*}=\frac{\varepsilon+\mu+\delta}{\eta \beta}\left[\frac{\eta \Lambda \beta(1-v)}{(\eta+\mu)(\varepsilon+\mu+\delta)}-\mu\right] \\
I^{*}=\frac{1}{\beta}\left[\frac{\eta \Lambda \beta(1-v)}{(\eta+\mu)(\varepsilon+\mu+\delta)}-\mu\right] \\
R^{*}=\frac{1}{\mu}\left[\Lambda v+\frac{\varepsilon}{\beta}\left(\frac{\eta \Lambda \beta(1-v)}{(\eta+\mu)(\varepsilon+\mu+\delta)}-\mu\right)\right]
\end{gathered}
$$

Thus, the characteristic equation becomes

$$
\left|J\left(E_{0}\right)-I \lambda\right|=\left[\begin{array}{cccc}
-\left(A_{1}+\lambda\right) & 0 & -\beta S^{*} & 0 \\
\beta I^{*} & -\left(A_{2}+\lambda\right) & \beta S^{*} & 0 \\
0 & \eta & -\left(A_{3}+\lambda\right) & 0 \\
0 & 0 & \varepsilon & -(\mu+\lambda)
\end{array}\right]=0
$$


where

$$
\begin{gathered}
A_{1}=\beta S^{*}+\mu \\
A_{2}=\eta+\mu \\
A_{3}=\varepsilon+\mu+\delta
\end{gathered}
$$

Hence, we have

$$
-(\mu+\lambda)\left[-\left(A_{1}+\lambda+\lambda\right)\left(A_{2}+\lambda\right)\left(A_{3}+\lambda\right)-\eta \beta S^{*}-\eta \beta^{2} S^{*} I^{*}\right]=0
$$

which further yields:

$$
\begin{aligned}
& -(\mu+\lambda)\left(A_{1} A_{2} A_{3}+A_{1} A_{2} \lambda+A_{1} A_{3} \lambda+A_{1} \lambda^{2}-\eta \beta A_{1} S^{*}+A_{2} A_{3} \lambda+A_{2} \lambda^{2}\right. \\
& \left.+A_{3} \lambda^{2}+\lambda^{3}-\eta \beta S^{*} \lambda-\eta \beta^{2} S^{*} I^{*}\right)=0
\end{aligned}
$$

Therefore,

$$
\begin{aligned}
& \lambda_{1}=-\mu, \\
& \lambda^{3}+\left(A_{1}+A_{2}+A_{3}\right) \lambda^{2}+\left(A_{1} A_{2}+A_{1} A_{3}+A_{2} A_{3}-\eta \beta S^{*}\right) \lambda \\
& +\left(A_{1} A_{2} A_{3}-\eta \beta A_{1} S^{*}-\eta \beta^{2} S^{*} I^{*}\right)=0 \\
& \lambda^{3}+a_{1} \lambda^{2}+a_{2} \lambda+a_{3}=0
\end{aligned}
$$

where

$$
\begin{gathered}
a_{1}=\left(A_{1}+A_{2}+A_{3}\right) \\
a_{2}=\left(A_{1} A_{2}+A_{1} A_{3}+A_{2} A_{3}-\eta \beta S^{*}\right) \\
a_{3}=\left(A_{1} A_{2} A_{3}-\eta \beta A_{1} S^{*}-\eta \beta^{2} S^{*} I^{*}\right)
\end{gathered}
$$

According to Routh-Hurwitz's criterion, all the roots of the equation will have negative real parts if the following conditions are met:

1) if $a_{1}>0, a_{2}>0, a_{3}>0$;

2) If $a_{1} a_{2}>a_{3}$.

If all these conditions are met, then it follows that all the eigenvalues satisfy the condition $|\arg (\lambda)|>\frac{\alpha \pi}{2}$. Hence, the Endemic Equilibrium Point is asymptotically stable.

\section{Numerical Simulation}

In this section, the predictor corrector method is applied to get the numerical solutions of system (3.6) [12]. We will propose two cases for the model (3.6) with various values of parameters. In the first case, $\Lambda=0.02755, \beta=0.09091$, $\mu=0.00875, \eta=0.125, \varepsilon=0.02755, \delta=0.125, v=0.7$ [13] and with initial conditions: $S(0)=500, E(0)=5, I(0)=20, R(0)=10$ (Estimated). In this case, $R_{0}=0.66<1$, then the disease free equilibrium is locally stable and the disease dies out. In the second case, $\Lambda=5, \beta=0.09091, \mu=0.00875$, $\eta=0.125, \varepsilon=5, \delta=0.125, v=0.7$ with same initial conditions, then $R_{0}=2.1>1$ which implies that the disease still persists and the endemic equilibrium is globally stable. Figure 2 shows the dynamics of Measles in a disease 
free equilibrium state. The curve obtained is an asymptote and it shows that the model would be asymptotically stable when $R_{0}<1$, which means that the disease will not invade the population rather it will die off with time as the decreasing asymptote curve does not intercept the horizontal axis.

Figure 3 reveals the variation of the exposed population with time when $R_{0}<1$. Since at disease free equilibrium, the susceptible human decreases as seen in the previous plot, hence the exposed class increases as the susceptible population decreases. Figure 4 shows that the infected humans are decreasing asymptotically with time. This implies that when $R_{0}<1$, an infected human would not infect up to an average person in the population. Therefore, the infected humans will recover continuously until no human would be infected in the population and hence, shows the stability of the disease-free equilibrium point. In Figure 5, we see an increasing graph for the recovered class when $R_{0}<1$. This shows that recovered class will increase over time as an infected humans does not infect up to an average susceptible human when the threshold parameter is less than 1 . Hence, the infected humans keep decreasing as most of them get recovered until no human is infected when $R_{0}<1$.

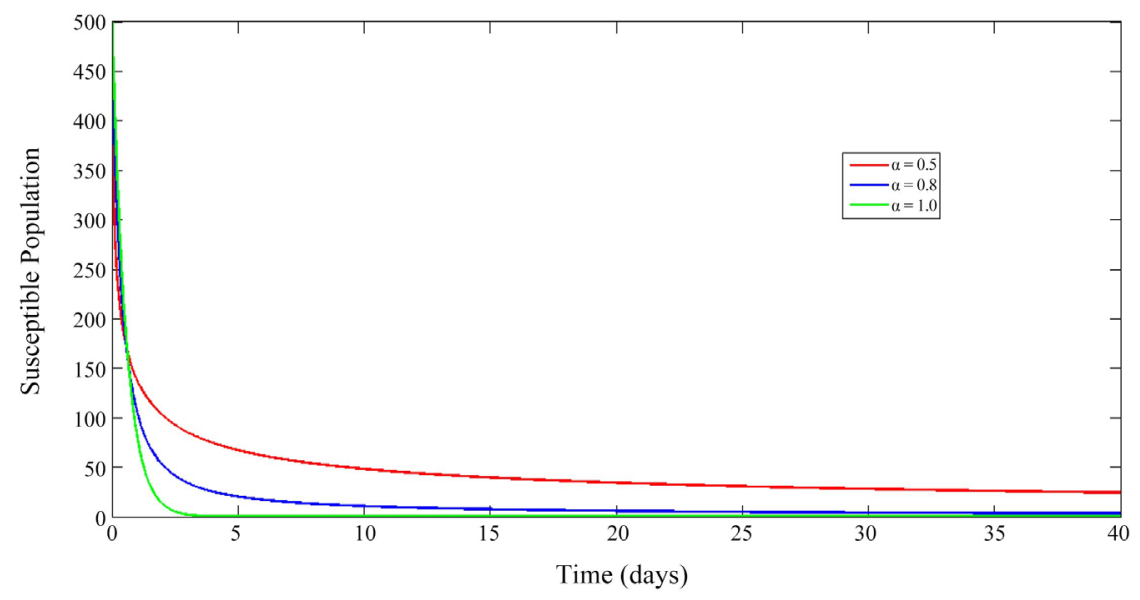

Figure 2. Dynamics of the susceptible population.

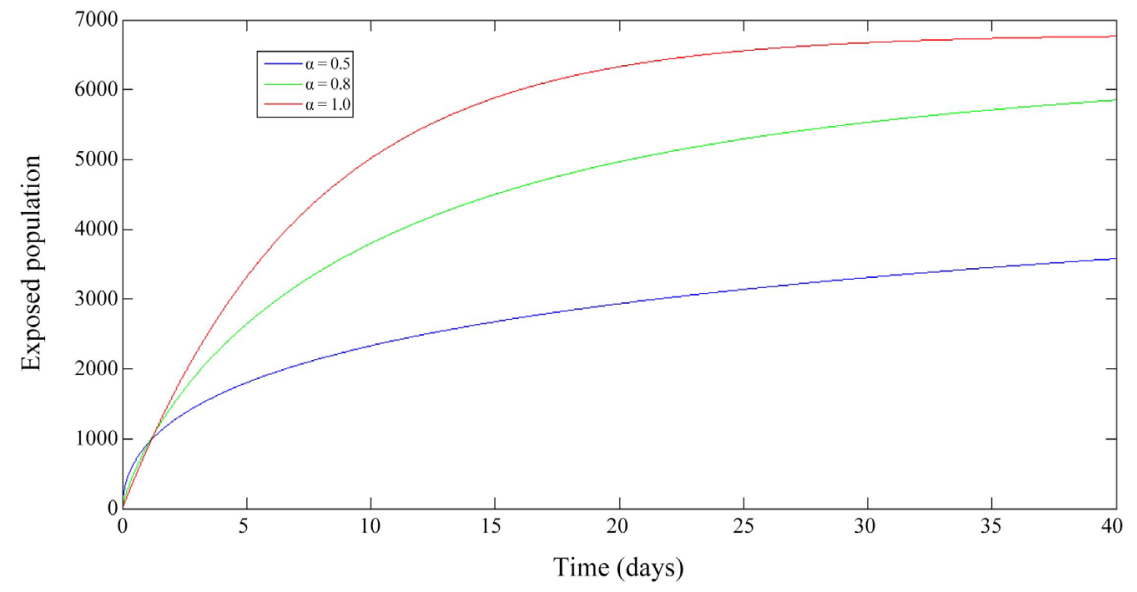

Figure 3. Dynamics of the exposed population with $R_{0}<1$. 


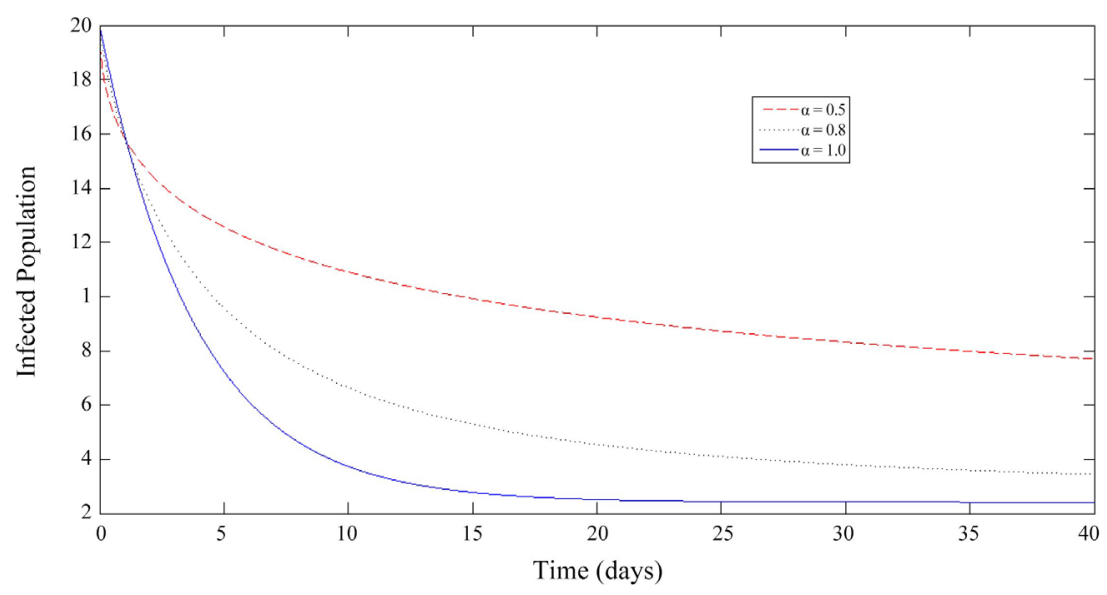

Figure 4. Dynamics of the infected population.

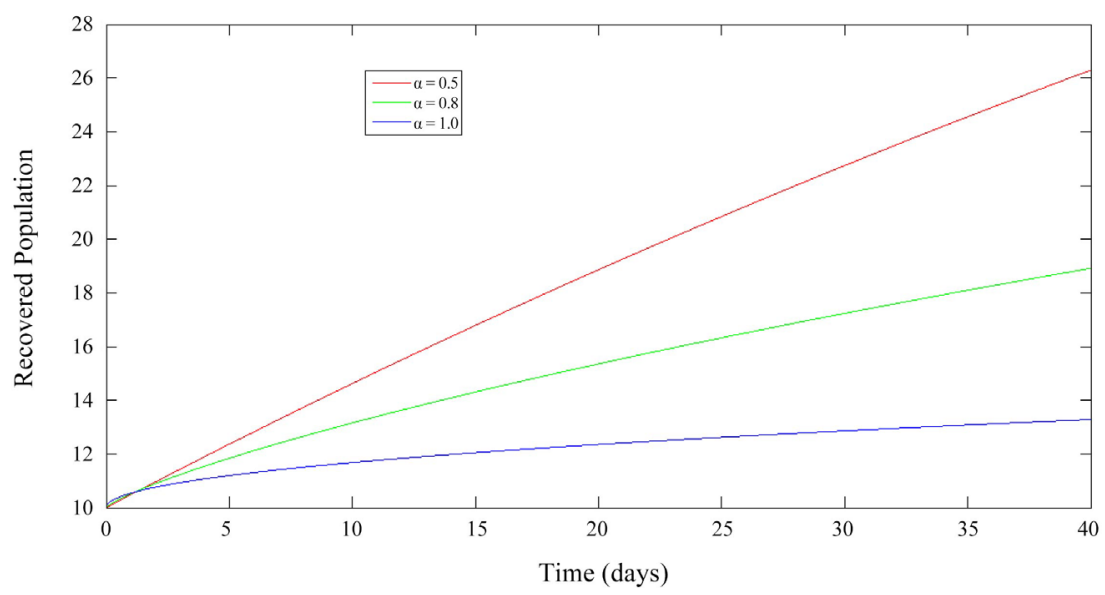

Figure 5. Dynamics of the recovered population with $v=0.7$.

\section{Conclusion}

A fractional order model for the transmission dynamics of measles with vaccination was presented. The model captures the causes of measles transmission and a possible way of preventing the disease transmission. It can be seen in the model that the spread of the disease largely depends on the contact rates with infected people within the population. It is seen that if the proportion of population that is immune increases, then the disease can no longer persist. Thus, if this can be achieved through mass vaccination or making vaccination compulsory, the disease can be prevented. The numerical simulations of the fractional order model with different values of $\alpha$ are performed by Caputo's derivative using the predictor corrector method of Adams-Bashforth Moulton type. The dynamics of the compartments have been shown in the graphs obtained. In addition, the results gave an insight that fractional order model is more suitable than its integer-order.

\section{Acknowledgements}

We wish to thank Prof. M. O. Oyesanya and my co-authors for their kind sug- 
gestions towards the success of this work.

\section{Conflicts of Interest}

The authors declare no conflicts of interest regarding the publication of this paper.

\section{References}

[1] Haq, F., Shahzad, M., Muhammad, S., Wahab, H.A. and Rahman, G. (2017) Numerical Analysis of Fractional Order Epidemic Model of Childhood Diseases. Discrete Dynamics in Nature and Society, 2017, Article ID: 4057089.

https://doi.org/10.1155/2017/4057089

[2] Wikipedia (2020) Measles. http://www.wikipedia.com/measles

[3] Goufo, E.F.D., Noutchie, S.C.O. and Mugisha, S. (2014) A Fractional SEIR Epidemic Model for Spatial and Temporal Spread of Measles in Metapopulations. Hindawi Publishing Corporation. Abstract and Applied Analysis, 2014, Article ID: 781028. https://doi.org/10.1155/2014/781028

[4] Nazir, G., Shah, K., Alrabaiah, H., Khalil, H. and Khan, R.A. (2020) Fractional Dynamical Analysis of Measles Spread Model under Vaccination Corresponding to Nonsingular Fractional Order Derivative. Advances in Difference Equations, No. 1, 1-15. https://doi.org/10.1186/s13662-020-02628-7

[5] Farman, M., Saleem, M.U., Ahmad, A.L. and Ahmad, M.O. (2018) Analysis and Numerical Solution of SEIR Epidemic Model of Measles with Non-Integer Time Fractional Derivatives by Using Laplace Adomian Decomposition Method. Ain Shams Engineering Journal, 9, 3391-3397. https://doi.org/10.1016/j.asej.2017.11.010

[6] Ahmed, E., EI-Sayed, A.M.A. and EI-Saka, H.A.A. (2006) On Some Routh-Hurwitz conditions for Fractional Order Differential Equations and Their Applications in Lorenz, Rossler, Chua and Chen Systems. Physics Letters A, No. 358, 1-4. https://doi.org/10.1016/j.physleta.2006.04.087

[7] Podlubny, I. (1999) Fractional Differential Equations. Academic Press, London.

[8] El-Sayed, A.M.A., Arafa, A.M.A., Kahlil, M. and Hassan, A. (2016) A Mathematical Model with Memory for Propagation of Computer Virus under Human Intervention. Progress in Fractional Differentiation and Applications, No. 2, 105-113. https://doi.org/10.18576/pfda/020203

[9] Van Den Driessche, P. and Watmough, J. (2002) Reproduction Numbers and Subthreshold Endemic Equilibria for Compartmental Models of Disease Transmission. Mathematical Biosciences, 180, 29-48. https://doi.org/10.1016/S0025-5564(02)00108-6

[10] Diekmann, J.A., Heesterbeek, P. and Metz, J.A.J. (1990) On the Definition and the Computation of the Basic Reproduction Ratio in Models for Infectious Diseases in Heterogeneous Populations. Mathematical Biosciences, 28, 363-382. https://doi.org/10.1007/BF00178324

[11] Heffernan, J.M., Smith, R.J. and Wahl, L.M. (2005) Perspectives on the Basic Reproduction Ratio. Journal of the Royal Society Interface, 2, 281-293. https://doi.org/10.1098/rsif.2005.0042

[12] El-Saka, H.A.A. (2014) The Fractional-Order SIS Epidemic Model with Variable Population Size. Journal of the Egyptian Mathematical Society, 22, 50-54. https://doi.org/10.1016/j.joems.2013.06.006 
[13] Kitengeso, R.E. (2016) Stochastic Modelling of the Transmission Dynamics of Measles with Vaccination Control. International Journal of Theoretical and Applied Mathematics, 2, 60-73. 